\title{
Searching in clutter: Visual attention strategies of expert pilots.
}

\author{
Melissa R. Beck ${ }^{1}$, Michael Trenchard ${ }^{2}$, Amanda van Lamsweerde ${ }^{1}$, Rebecca R. Goldstein ${ }^{1}$, Maura Lohrenz ${ }^{3}$ \\ ${ }^{1}$ Louisiana State University \\ ${ }^{2}$ Naval Research Laboratory \\ ${ }^{3}$ Volpe National Transportation Systems Center
}

Clutter can slow visual search. However, experts may develop attention strategies that alleviate the effects of clutter on search performance. In the current study we examined the effects of global and local clutter on visual search performance and attention strategies. Pilots and undergraduates searched for an elevation marker in charts of high, medium, and low global clutter. The target was in a low or high local clutter region of the chart or it was absent. High global and local clutter slowed search performance for both pilots and undergraduates. Pilots were more accurate but slower. Pilots' search strategies differed from undergraduates in the following ways: they had more conservative criteria for responding target absent and spent more time processing the information within each fixation. Pilots and undergraduates used a coarseto-fine search strategy in which, as the trial progressed, fixation durations increased and saccade distance decreased.

\section{INTRODUCTION}

Many everyday tasks require searching for a target of interest among a lot of distracting visual information. The amount of clutter (the distracting information) is one factor that can affect search performance (Lohrenz et al., 1997; Schons \& Wickens, 1993). For example, pilots use aeronautical charts to plan their flights and while in flight. These charts are often very cluttered, making the target of search difficult to find. In the current paper we address the following questions:

- How does the overall clutter in a chart affect pilots' ability to find a target quickly and accurately?

- Is performance related to the amount of clutter near the target location?

- Do visual attention search strategies (as measured by tracking eye movements) change depending on the amount of clutter in the chart?

- Does the expertise of pilots, as compared to non-pilots, lead to different visual search strategies?

Our previous work demonstrated that search time in a complex search task (finding an elevation marker in a chart) increased as the amount of clutter in the chart increased (Beck, Lohrenz \& Trafton, 2010). We used a Color-Clustering Clutter (C3) algorithm designed to quantify the amount of clutter in visual displays. $\mathrm{C} 3$ is a good predictor of subjective ratings of clutter (Lohrenz, Trafton, Beck, \& Gendron, 2009) and visual search performance (Beck, Lohrenz, \& Trafton, 2010). Furthermore, when participants searched through charts that varied in the amount of global clutter (the clutter of the whole chart) and the amount of local clutter (the amount of clutter surrounding the target), search reaction time (RT) was slower as the amount of global clutter increased. This effect was strongest when the target was in a high local clutter region. In addition, eye movements indicated that the increase in RT for higher levels of global clutter was caused by an increase in the duration and number of fixations that occurred before the target was found (Beck, Lohrenz, \& Trafton, 2010). The increase in the number of fixations indicates that increasing global clutter increases the number of areas in the charts competing with the target for attention.

Global clutter may also affect the attentional strategies participants employ. Given that participants are able to subjectively evaluate the amount of clutter in a visual display (Lohrenz, Trafton, Beck, \& Gendron, 2009), they may be able to use this information to guide the allocation of visual attention. By analyzing the scan paths during visual search in cluttered displays, we determined that participants tend to avoid cluttered regions early in search and move to more cluttered areas as search progresses (Lohrenz \& Beck, 2010). Furthermore, visual attention strategies may differ for charts of high versus low clutter. Searching in complex displays has been associated with a coarse-to-fine search strategy: fixation durations increase and saccade distance decreases as search progresses (Over, Hooge, Vlaskamp, \& Erkelens, 2007). A coarse-to-fine search strategy involves searching initially for 'low hanging fruit', followed by a more effortful search when the target is not located. We also found that participants used a coarse-to-fine search strategy when searching for an elevation marker in charts of all levels of clutter (Beck \& Lohrenz, 2011). Interestingly, the amount of global clutter (i.e., average clutter of the entire chart) affected fixation durations more than saccade distance. When global clutter was low, fixation durations were shorter, but saccade distance was similar to that in more cluttered charts. This suggests that higher levels of global clutter increase the time needed to process information within a given region, but do not change the size of the region attended (the attentional window) during the fixation. Therefore, a coarse-to-fine search strategy was found for charts of all levels of clutter and less cluttered charts did not lead to a broader focus of attention.

In the current research we examined how clutter affects performance and search strategies for participants with expertise in tasks related to the chart search task (pilots) versus participants without expertise (undergraduates). All of the previous research we have discussed has used undergraduates as the participants. These undergraduates 
likely have little to no experience with the charts or expertise in tasks that require efficient allocation of visual attention (e.g., flying an F/A-18 jet). Therefore, it is unknown if expertise will lead to different effects of clutter on visual search performance and attention strategies. Research has demonstrated that experts perform better on tasks within their area of expertise because they use a more efficient allocation of visual attention (e.g., Christensen et al., 1981; Ferrari, Didierjean, \& Marmèche, 2008; Jarodzka, Scheiter, Gerjets, \& van Gog, 2010; Myles-Worsley, Johnston, \& Simons, 1988). Therefore, experts' visual search performance may be less affected by clutter and experts may show more efficient visual search strategies.

\section{METHOD}

\section{Participants}

Thirty-one undergraduates ( 22 female) with an average age of 20 (range: $18-27, \mathrm{SD}=1.7$ ) participated in this experiment for course credit. Thirty-one F/A-18 pilots (28 male) in the U.S. Navy with an average age of 30 (range: 25$38, \mathrm{SD}=4.4$ ) also participated in this experiment. The pilots had an average of 1390 flight hours (range: $350-2750, \mathrm{SD}=$ 678 ) and 242 combat flight hours (range: $0-750, \mathrm{SD}=202$ ). About half of the pilots were flight instructors (42\%).

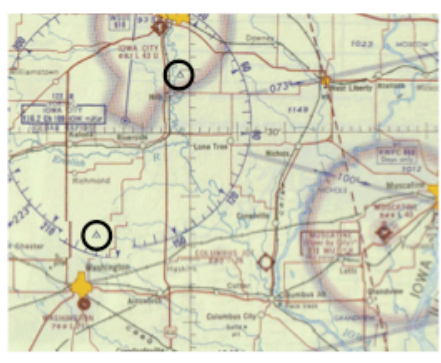

Low Global Clutter

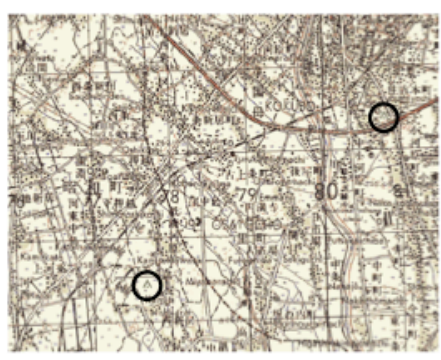

High Global Clutter

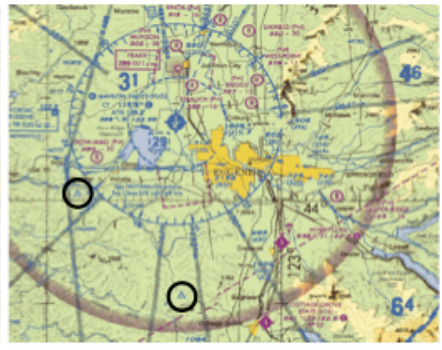

Medium Global Clutter

\begin{tabular}{|c|c|c|c|}
\hline & $\mathrm{L}$ & $\mathrm{M}$ & $\mathrm{H}$ \\
\hline Global & 2.9 & 6.1 & 8.3 \\
\hline $\begin{array}{c}\text { Low } \\
\text { Local }\end{array}$ & 1.7 & 4.6 & 7.0 \\
\hline $\begin{array}{c}\text { High } \\
\text { Local }\end{array}$ & 4.5 & 7.3 & 9.2 \\
\hline
\end{tabular}

Average C3 Values
Figure 1: Examples of the low, medium and high global clutter charts. Black circles indicate the location of high and low local clutter targets. In the experiment, only one target was present or no target was present. The black circles were not present in the experiment. On the bottom right is a table of the average global, low local, and high local C3 clutter values for the low $(\mathrm{L})$, medium $(\mathrm{M})$, and high $(\mathrm{H})$ global clutter charts.

\section{Apparatus}

An Eyelink II (SR Research) head-mounted eye tracker was used to collect eye movement data from the undergraduate participants. An Eyelink 1000 (SR Research) desk-mounted eye tracker was used to collect eye movement data from the pilots. The Experiment Builder (SR Research) program was used to create the experiment for both groups of participants.

\section{Stimuli}

Seventy-two base charts ( 24 each of low, medium, and high global clutter) were used in this experiment (see Figure 1). Three different versions of each base chart were created: the target was either placed in a region of high or low local clutter or the target was absent. The same target was used in each chart, with the exception that the color of the target varied so that the target color was similar to other colors in the chart. Participants saw each of the 72 charts once with the target either in the low or high local clutter region or the target was absent. Within each bin of global clutter, participants saw eight high-local-clutter charts, eight lowlocal-clutter charts, and eight target-absent charts. The presentation order was randomly determined for each participant. Global and local clutter ratings (see Figure 1) were determined using the $\mathrm{C} 3$ model (Lohrenz, Trafton, Beck, \& Gendron, 2009). For the pilots the charts were $881 \times 690$ pixels and $24 \times 19 \mathrm{~cm}$ and for the undergraduates they were $740 \times 580$ and $29 \times 19 \mathrm{~cm}$.

\section{Procedure}

Participants determined whether a target was present or absent in each chart by pressing one of two buttons on the right button pad of a game controller. If they responded that the target was present, the chart would disappear, followed by a screen that instructed them to click on the target with the mouse. Then the chart appeared on the screen again, at which point they used their mouse to click on the location of the target. If the participants indicated that the target was absent, the trial would end and the next trial would begin. If no response was made within one minute, the trial would end. Participants were informed that if there was a target on the map, there would be only one and participants were encouraged to respond as quickly as possible.

\section{RESULTS}

Responses were coded as accurate (a response was given and it was correct), inaccurate (a response was given and it was inaccurate), or timeouts (no response was given within the one minute time limit). Accuracy was examined for target present and target absent trials so that hits, false alarms, correct rejections and misses could be assessed. $3 \times 3 \times 2$ mixed model ANOVAs were conducted with global clutter (high, medium, low) and target presence (high, low, absent) as within-subjects factors and expertise (pilots, non-pilots) as a between-subjects factor for the following measures: accuracy, timeouts, reaction time on accurate trials, number of fixations on accurate trials, and fixation durations on accurate trials.

\section{Accuracy}

Accuracy was calculated by dividing the number of accurate trials by the number of trials for which a response 
was given within the one-minute time limit. Data from two pilots was excluded because, in at least one condition, all trials were timeouts. There were main effects for global clutter, $F(2$, $232)=91.84, M S=1.9, p<.001$, target presence, $F(2,232)=$ $155.26, M S=4.7, p<.001$, and expertise, $F(1,58)=12.54$, $M S=1.0, p=.001$ (see Figure 2). There were also significant interactions between global clutter and target presence, $F(4$, 232) $=37.18, M S=.6, p<.001$, global clutter and expertise, $F(2,232)=7.44, M S=.16, p=.001$, and target presence and expertise, $F(2,232)=18.28, M S=.55, p<.001$.

Pilots were significantly more accurate (more hits and fewer misses) than the undergraduates on the high global clutter target present trials for both low and high local clutter and on the medium global/ high local clutter trials (all $p \mathrm{~s}<$ $.05)$. Pilots and undergraduates did not differ for any of the other trial types.

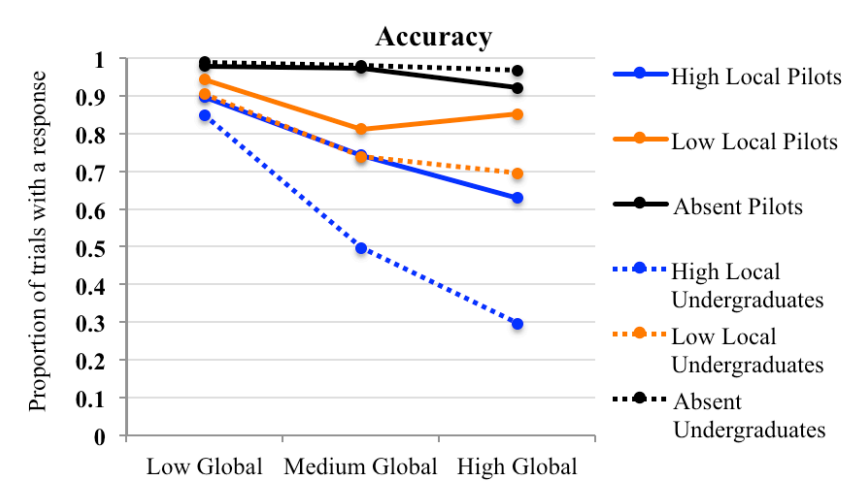

Figure 2: Proportion of trials in which an accurate response (hits for target present trials and correct rejections for target absent trials) was given within the one-minute time limit.

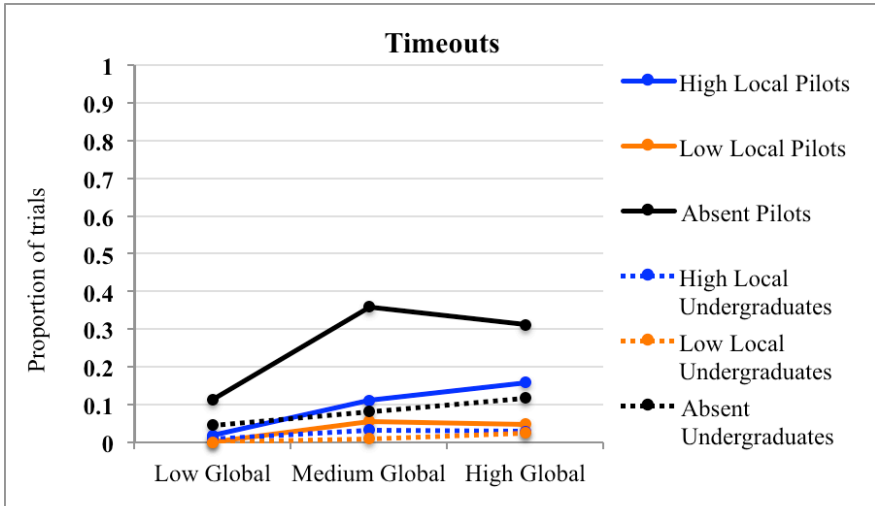

Figure 3: Proportion of all trials in which no response was given within the one-minute time limit.

\section{Timeouts}

An analysis of the proportion of all trials for which no response was given within the one-minute time limit revealed main effects for global clutter, $F(2,240)=40.86, M S$ $=.41, p<.001$, target presence, $F(2,240)=41.75, M S=1.12$, $p<.001$, and expertise, $F(1,60)=24.19, M S=.1 .12, \mathrm{p}<.001$ (see Figure 3). There were also significant interactions between global clutter and target presence, $F(4,240)=6.61$,
$M S=.06, p=.001$, global clutter and expertise, $F(2,240)=$ $15.87, M S=.16, p<.001$, and target presence and expertise, $F(2,240)=11.21, M S=.30, p<.001$.

Pilots had more timeouts than undergraduates on the target absent trials for both high and medium global clutter (all $p$ s <.05). Therefore, pilots were more conservative with giving a "target absent" response. Pilots also had more timeouts on the high global/high local, medium global/high local, and medium global/low local trials (all $p \mathrm{~s}<.05$ ).

\section{Reaction Time}

Reaction time, number of fixations, and fixation duration analyses included only accurate trials. Data from seven undergraduates and two pilots was excluded because there were no accurate trials in at least one of the conditions.

There were main effects for global clutter, $F(2,204)$ $=104.42, M S=3350.9, p<.001$, target presence, $F(2,204)=$ $562.15, M S=31925.2, p<.001$, and expertise, $F(1,51)=$ 25.63, $M S=4830.5, p<.001$ (see Figure 4). There were significant interactions between global clutter and expertise, $F(2,204)=6.16, M S=146.9, p=.003$, and between target presence and expertise, $F(2,204)=19.6, M S=1113.4, p<$ .001 . Pilots were slower to respond for target absent trials at all levels of global clutter and slower on the high global/high local, medium global/high local, and low global/low local target present trials (all $p s<.05$ ).

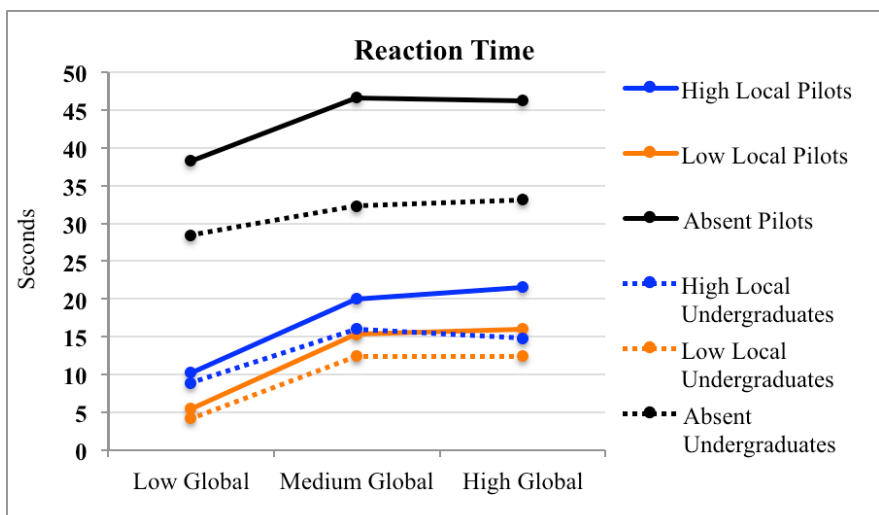

Figure 4: Reaction time for accurate trials.

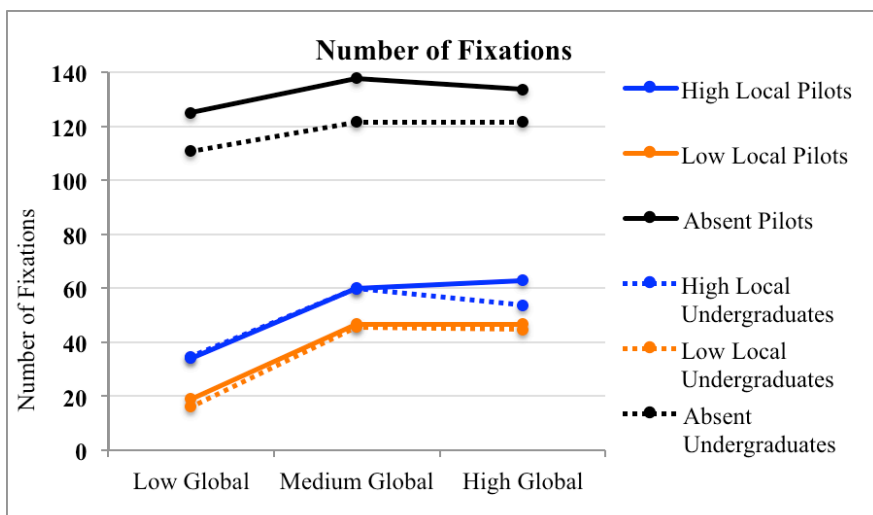

Figure 5: Number of fixations for accurate trials. 


\section{Number of Fixations}

There were main effects for global clutter, $F(2,204)$ $=90.01, M S=24054.8, p<.001$, and target presence, $F(2$, $204)=473.73, M S=356639.9, p<.001$, but not for expertise, $F(1,51)=1.8, M S=4595.8, p=.18$ (see Figure 5). The only significant interaction was between global clutter and target presence, $F(4,204)=7.1, M S=1519.3, p<.001$.

\section{Fixation Duration}

There were main effects for global clutter, $F(2,204)$ $=16.0, M S=13675.7, p<.001$, target presence, $F(2,204)=$ 18.6, $M S=29583.7, p<.001$, and expertise, $F(1,51)=35.7$, $M S=555841.7, p<.001$ (see Figure 6). There were significant interactions between global clutter and expertise, $F(2,204)=8.1, M S=6882.6, p<.001$, and between global clutter and target presence, $F(4,204)=3.1, M S=2430.3, p=$ .02 . Pilots' fixations were significantly longer than the undergraduates' for all trial types (all $p \mathrm{~s}<.05$ ) except for the low global clutter/ low local clutter target present trials.

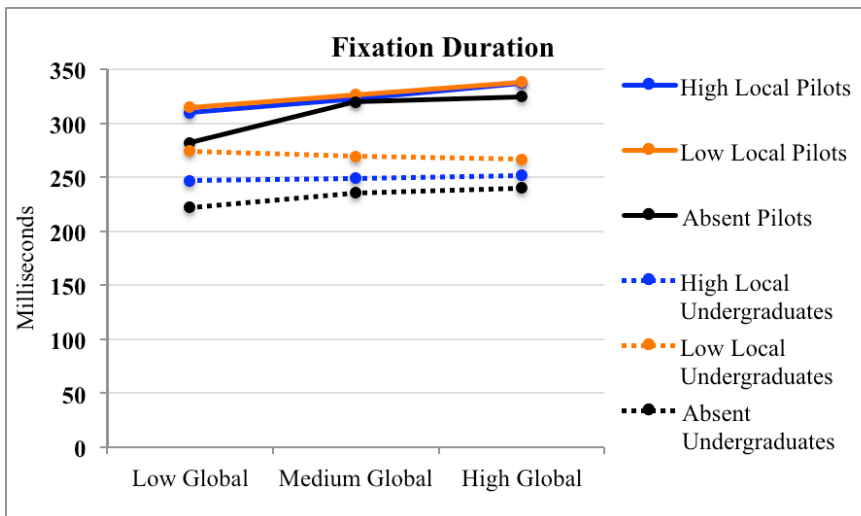

Figure 6: Fixation duration for accurate trials.

\section{Coarse-to-Fine}

Fixation duration and saccade distance were examined across the first 40 fixations of all trials (see Figure 7). The first two fixations were excluded from the analysis because they tend to show a different pattern than the remaining fixations. The charts the pilots saw were a higher resolution, so for comparison purposes, the pixel distance data for the pilots was scaled to match the undergrad data. For the pilots, fixation durations increased as fixation number increased for low (slope $=1.04)$, medium (slope $=2.03$ ) and high (slope $=2.12$ ) global clutter. Although slopes were shallower for the undergraduates, fixation durations also increased across fixation number for low (slope $=0.31$ ), medium (slope $=0.75$ ), and high global clutter (slope $=0.77$ ). In line with a coarse-to-fine search strategy, as fixation durations increased across fixation number, saccade distance decreased. For pilots, saccade distance decreased across fixation number for low (slope $=-1.34$ ), medium (slope = 1.36), and high (slope $=-1.36$ ) global clutter and for undergraduates, saccade distance decreased across fixation number for low (slope $=-1.56$ ), medium (slope $=-1.46$ ), and high (slope $=-1.46)$ global clutter.

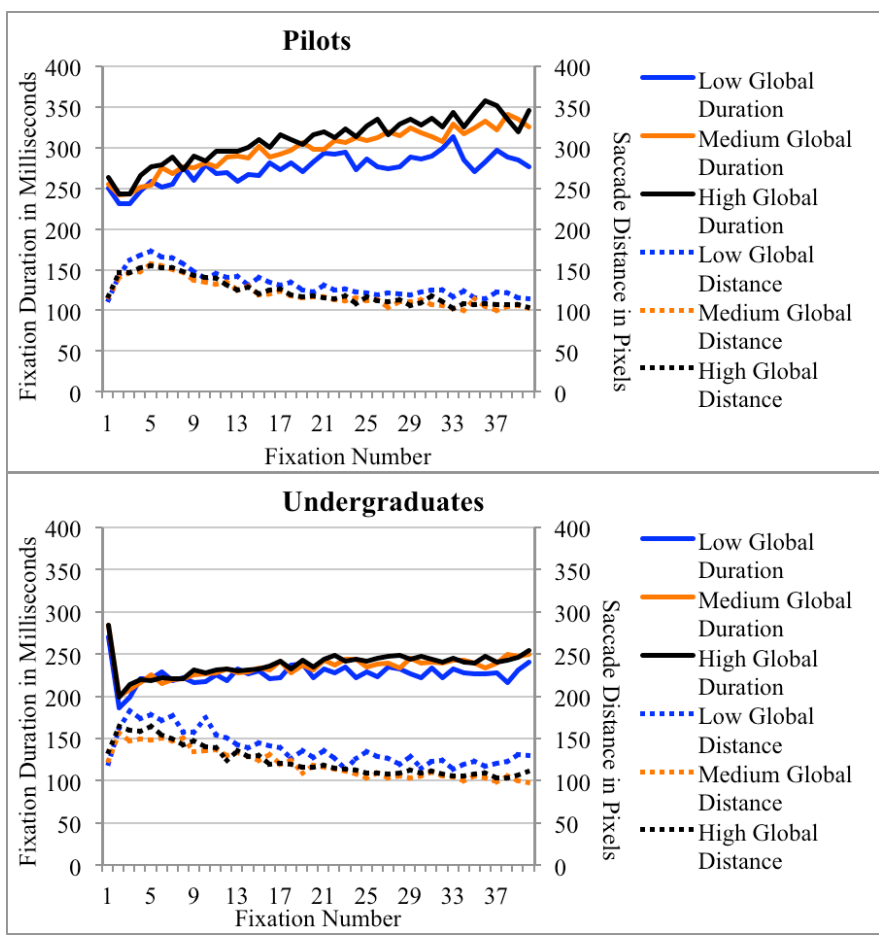

Figure 7: Fixation duration (milliseconds) and saccade distance (pixels) for the first 40 fixations.

\section{DISCUSSION}

We replicated previous research (Beck, Lohrenz, \& Trafton, 2010) by showing that global and local clutter slow search performance and this caused an increase in the number of fixations and fixation durations. In addition, global and local clutter impaired search accuracy such that performance was poorest when both local and global clutter were high. However, the effects of local and global clutter on accuracy were weaker for pilots than for undergraduates.

Undergraduates showed a greater decrease in accuracy as global and local clutter increased. Specifically, pilots were more accurate than undergraduates on the more difficult target present trials: when there was medium or high global clutter and high local clutter. In addition to being more accurate on the more difficult target present trials, pilots also had longer RTs and more timeouts on these trials suggesting that they were using a more conservative criterion when responding. Performance on the target absent trials also suggests a more conservative response criterion. Pilots were not more accurate on the target absent trials when a response was given.

However, they had longer RTs and increased timeouts on target absent trials, suggesting they had a more conservative response criterion for deciding that the target was absent.

Pilots were slower to respond than undergraduates on the target absent trials and on the target present trials with high global clutter (for both low and high local clutter) and medium global with high local clutter. Longer RTs were not caused by pilots making more fixations than undergraduates. Rather, pilots' fixation durations were longer for most trial types. This suggests that pilots were slower because they were spending more time processing the information within each fixation. 
Although this generally improved accuracy for the pilots on the target present trials, it also resulted in slower performance.

The coarse-to-fine analysis revealed that both pilots and undergraduates were using a coarse-to-fine search strategy: fixations durations increased and saccade distance decreased as the trial progressed. However, pilots had a stronger tendency for fixation duration to increase. This, combined with the overall longer fixation durations for pilots, further suggests that pilots were processing the information within each fixation more fully than undergraduates. This was particularly the case for medium and high global clutter charts. Although fixation durations were longer for pilots, saccade distances were not. Therefore pilots and undergraduates were processing information within the same size attentional window, but pilots spent more time processing the information.

Overall, pilots performed better, but were slower to respond, in this experiment. Pilots exhibited a more conservative stopping rule and spend more time processing information within a given fixation. This suggests that expertise may lead to a more effortful and conservative approach to the target search task. Interestingly, this strategy does not always lead to improved performance. Particularly on target absent trials, pilots' search was slower, but there were more timeouts. Therefore, pilots may be able to improve performance by changing their response criterion.

Furthermore, training plans for novices should involve instruction on using more effortful and conservative attention strategies for the more difficult search types (high and medium global clutter and target absent trials).

The instructions provided for a search task may influence performance and attention allocation. For example, the current results are not consistent with a recent finding by Godwin et al (2012). They looked at search behavior for experienced and inexperienced Improvised Explosive Device (IED) searchers. Unlike the current results, experienced searchers responded "target absent" more frequently and responded more quickly than inexperienced searchers. This task may involve greater risk for false alarms than was present in the current search task. In the current study participants were told to respond as quickly as possible, however, no emphasis was placed on the type of response that was the most critical. It appears that pilots viewed "misses" as more negative than timeouts, while the undergraduates were more willing to risk a "miss" by giving a target absent response after searching for less time. Providing specific instructions to pilots and undergraduates that stressed the importance of responding correctly, but before the time limit, may have lead to more similar behavior between the two groups.

The main goal of the current research was to see if the effects of global and local clutter on search performance and search strategies would replicate in a sample of pilots. A secondary goal was to directly assess differences between participants with and without expertise related to the task. Ideally, in order to make conclusions about the effects of expertise on search performance and visual attention strategies, we would need a control group that differed from the pilots only in their level of expertise. Unfortunately, this was not the case for the undergraduates in this study. For example, the pilots were on average older than the undergraduates. Given the correlations between age and accuracy (undergraduates $=.04$; pilots $=-.13$ ) and age and RT (undergraduates $=.22$; pilots $=.15$ ), it is possible that age played a role in the results found in the current study. In addition, group differences in search performance and strategies in the current data may have been influenced by other factors as well (e.g., gender, education level, motivation, etc.) Our future research goals include replicating the current study with a control group that more closely matches the pilots in factors other than expertise that may influence search performance and strategies.

This research is an important first step in determining the differences in visual attention strategies and visual search performance between novices and experts. Ultimately this research can lead to improvements in mission planning, training, and chart design.

\section{ACKNOWLEDGEMENTS}

This research was funded by NRL Broad Agency Announcement grant 0748,554741 to the first author and Program Element $602435 \mathrm{~N}$ by the NRL 6.2 Base Program to the second author.

The authors thank the students at the Louisiana State University (LSU) Psychology Department and the pilots and navigators at NAS-Oceana, VA who participated in these experiments. Further, the authors would like to thank LCDR Joseph "Krusty" Cuba (Operations, Commander Strike Fighter Wing Atlantic) for his extensive support including the recruitment of participants at NAS-Oceana.

\section{REFERENCES}

Beck, M.R., Lohrenz, M.C., \& Trafton, J.G. (2010). Measuring Search Efficiency in Complex Visual Search Tasks: Global and Local Clutter. Journal of Experimental Psychology: Applied, 16(3), 238-250.

Christensen, E. E., Murry, R. C., Holland, K., Reynolds, J., Landay, M. J., \& Moore, J. G (1981). The effect of search time on perception. Diagnostic Radiology, 138, 361-365.

Ferrari, V., Didierjean, A., \& Marmèche, E. (2008). Effect of expertise acquisition on strategic perception: The example of chess. The Quarterly Journal of Experimental Psychology, 61 (8), 1265-1280.

Godwin, H. J., Liversedge, S. P., Kirkby, J. A., Cornes, K., Boardman, M., \& Donnelly, N. (2012, May). The influence of experience upon threat assessment and visual search in complex scenes. Poster presented at the annual meeting of the Vision Sciences Society, Naples, FL.

Jarodzka, H., Scheiter, K., Gerjets, P., \& van Gog, T. (2010). In the eyes of the beholder: How experts and novice interpret dynamic stimuli. Learning and Instruction, 20 (2), 146-154.

Lohrenz, M.C. \& Gendron, M.L. (2008). A 3D clustering algorithm to model clutter in electronic geospatial displays. Journal of Management and Engineering Integration, 1(2), 83-88.

Lohrenz, M.C., Trafton, J.G., Beck, M.R., \& Gendron, M.L. (2009). A model of clutter for complex, multivariate, geospatial displays. Human Factors, 51(1), 90-101.

Lohrenz, M., Trenchard, M., Myrick, S., Van Zuyle, P., \& Fechtig S. (1997). Optimizing cockpit moving-map displays for enhanced situational awareness. Chapter 13 in Situational awareness in the tactical air environment: Augmented proceedings of the Naval Air Warfare Center's first annual symposium, 363-387. CSERIAC, Wright-Patterson AFB, OH. July.

Myles-Worsley, M., Johnston, W. A., \& Simons, M. A. (1988). The influence of expertise on x-ray image processing. Journal of Experimental Psychology: Learning, Memory, and Cognition, 14 (3), 553-557.

Over, E.A.B., Hooge, I.T.C., Vlaskamp, B.N.S., \& Erkelens, C.J. (2007) Coarse-to-fine eye movement strategies in visual search. Vision Research, $47,2272-2280$.

Schons, V. \& Wickens C. (1993). Visual separation and information access in aircraft display layout. University of Illinois Institution of Aviation Technical Report ARL-93-7/NASA-A3I-93-1, Savoy, IL. 